Philosophie ANTIQUE

\section{Philosophie antique}

Problèmes, Renaissances, Usages

17 | 2017

Platon et la politique

\title{
Wilhelm KROLL, Discours sur les oracles chaldaïques
}

\section{Adrien Lecerf}

\section{OpenEdition}

\section{Journals}

Édition électronique

URL : https://journals.openedition.org/philosant/299

DOI : 10.4000/philosant.299

ISSN : 2648-2789

Éditeur

Éditions Vrin

\section{Édition imprimée}

Date de publication : 1 novembre 2017

Pagination : 227-230

ISBN : 978-2-7574-1807-9

ISSN : $1634-4561$

\section{Référence électronique}

Adrien Lecerf, « Wilhelm kroLl, Discours sur les oracles chaldaïques », Philosophie antique [En ligne], 17 | 2017, mis en ligne le 01 novembre 2018, consulté le 02 décembre 2022. URL : http:// journals.openedition.org/philosant/299; DOI : https://doi.org/10.4000/philosant.299

\section{(c) (i) $\odot$}

Creative Commons - Attribution - Pas d'Utilisation Commerciale - Pas de Modification 4.0 International - CC BY-NC-ND 4.0

https://creativecommons.org/licenses/by-nc-nd/4.0/ 
project as the product of the first philosophy, and offers some very interesting remarks on the first passage above showing how metaphysics subsumes logic and takes its place in the old tripartite Hellenistic division.

The third chapter 'The objects of metaphysics' shows how the results of this analysis of the scope and tasks of metaphysics can be applied to explain in a unified way the variety of objects this science deals with, from the most widely dispersed non-substantial being to the most unique first unmoved mover. Since the link between the higher and lower ontological orders is construed as explanatory/causal, this leads us to the discussion of the mechanism of the three types of causation: the causation of substance with relation to non-substantial beings, the causation of form with respect to substance, and the causation of the separate form, the first unmoved mover, in relation to the human intellect and to the world as a whole (theology). In all three cases, the 'bridge' principle of the causality of the maximum described above is deployed in order to provide a link between the different ontological levels. This principle works to explain the causal priority of substance over non-substantial beings, without making the latter the mere tropes of the former: to ensure this, Alexander develops the concept of a kind of being that is 'intermediate' between the substance and the accident that is fully dependent on a substance: the corresponding relation is that of 'strong' homonymy $\alpha \dot{\alpha}$ ' 'हv $v$ ć and $\pi \rho \grave{\varsigma} \varsigma \check{\varepsilon} v$, between the substance and the related non-substances. The same principle of causal priority works also in the case of form-substance over composite substance. This section is most interesting in light of the recent discussions of Alexander's essentialism vs attributivism of earlier Peripatetics (Andronicus, Boethus). Guyomarc'h argues that Alexander's account of form-substance can in fact accommodate Boethus' view of form as a quality as a partial or incomplete account to the extent that it agrees with the causal priority of substance. Particularly interesting and apt is Guyomarc'h's explanation (even if written out in broad strokes) of how the causality of the maximum works in Alexander's noetics, to explain the relation between the divine intellect and human intellect. This argument in Alexander's De anima $(88,26-89,11)$ is often taken by readers to be a Platonic influence, and showing its roots in Alexander's considered interpretation of Aristotle's Metaphysics is thus a very important result.

On the whole, the book is excellent, very rich, thought provoking, engaging, eminently readable. Guyomarch has not only worked with great care through many little studied texts of Alexander and provided a convincing account of Alexander's overall argument in the Metaphysics commentary. He has also done an exemplary work of finding the place of this argument with all its complex developments in modern discussions of Aristotle's Metaphysics, with which he is fully engaged throughout the book, making many interesting suggestions and shedding new light on many old and controversial topics. This is a very significant contribution to the field, and a must read for all students of Alexander and Aristotle's Metaphysics.

Inna Kupreeva University of Edinburgh

Wilhelm KROLL, Discours sur les oracles chaldaïques, traduction par H. D. Saffrey, Paris, Vrin, 2016 (Textes et traditions, 28), 126 p., ISBN : 978-2-7116-2746-2.

(Toutes références données aux pages de la traduction, et non de l'œuvre originale traduite).

Plus d'un siècle après sa première parution (Breslau 1894), la dissertation latine de W. Kroll (ci-après : K.), ouvrage majeur qui fit accéder l'étude des Oracles chaldaïques (ci-après : $O C$ ) à un statut scientifique, trouve une seconde naissance à travers la 
traduction donnée par H. D. Saffrey (ci-après : S.). Cette entreprise, aussi bien, constitue un hommage d'un savant d'une longévité exceptionnelle à un autre, qui publia très jeune (à vingt-cinq ans) cette œuvre pionnière.

Dans le style concis et clair dont il est coutumier, S. s'explique dans un Avertissement (p. VII-XII) sur les raisons qui l'ont motivé ainsi que sur sa méthodologie : « La lecture du latin étant devenue difficile..., j'ai cru me rendre utile en traduisant ce texte en français. De plus, comme il s'agit non pas seulement d'une énumération de fragments, mais surtout d'un discours raisonné sur ce que K. croyait pouvoir dire de cet ouvrage perdu, j'ai cru bon d'imprimer en caractères de corps différents le discours lui-même et les fragments venant des auteurs néoplatoniciens en donnant les références aux éditions modernes et en ajoutant les numéros donnés par Éd. des Places dans son édition des $O C$. Les pages de $\mathrm{K}$. sont données dans le corps du texte en caractères gras ».

S. s'appuie donc sur la double nature du texte de K. - monographie, mais aussi édition avant la lettre -, mise en évidence au moyen d'une mise en page complexe et pourtant irréprochable. Les fragments des $O C$, signalés par un retrait typographique, sont donnés successivement en grec et dans une traduction reprise pour l'essentiel (mais souvent avec de notables modifications) des éditions de référence qui n'existaient pas du temps de K. Les $O C$ identifiés depuis 1894 sont rappelés par $\mathrm{S}$. (p. X-XII). De très utiles sous-titres ont été insérés afin de rendre le propos plus aisé à suivre, et des références philologiques obsolètes ont été supprimées : S. signale ses interventions - peu nombreuses - dans le texte de K. par des crochets. Un Index (p. 113-123) recense l'ensemble des sources. Enfin, une traduction d'un court article de K. en allemand, sorte de synthèse de sa dissertation latine et déjà jointe à la réimpression de celle-ci (Hildesheim 1962), est donnée.

S. a ainsi fourni un précieux instrument de travail à qui souhaite prendre connaissance du travail de K. Mais qu'en est-il de la valeur intrinsèque de celui-ci, après tant d'années, et alors qu'est parallèlement publiée la très bonne introduction du professeur $\mathrm{H}$. Seng (Un livre sacré de l'Antiquité tardive : les Oracles chaldaïques, Turnhout 2016) ?

On doit tout d'abord constater que le livre de K. constitue toujours, à travers sa reprise dans l'édition de référence de Des Places (Paris 1971), la base éditoriale sur laquelle est fondée notre connaissance des $O C$. Quiconque lira le livre dans l'ordre (ou se reportera à la concordance annexée à l'édition de Des Places) en considérant la succession des renvois ajoutés par S. constatera en effet que l'ordre de Des Places était déjà celui de K., le savant français n'ayant fait, pour l'essentiel, que déplacer les expressions isolées à la fin de son édition et introduire une section de Dubia, dont la pertinence a été mise en doute par M. Tardieu (dans la réédition de H. Lewy, Chaldean Oracles, Paris $1978^{2}$, p. 680). L'ordre thématique imposé aux fragments par K. n'est pas totalement satisfaisant, mais il est peu probable qu'on puisse le modifier sensiblement à l'avenir : peut-être un travail minutieux sur leurs acolouthies et groupements pourra-t-il faire progresser la recherche. Par acquit de conscience, on pourrait également tester l'hypothèse de l'influence des $O C$ sur l'ordre systématique adopté par les néoplatoniciens, par exemple dans la Théologie platonicienne, comme le suggère S. lui-même (p. virI).

La forme choisie par K., au reste, a aussi plusieurs avantages : grâce aux contextes qu'il cite, il est parfois loisible de constater qu'une coupure quelconque intervient entre plusieurs morceaux d'oracles que Des Places édite pourtant, de façon contestable, sous un même numéro de fragment (par ex. OC $60 ; 160$ ). Des différences d'attribution, également, apparaissent : ainsi l'OC 71, p. 52, est-il prononcé par $\tau \iota \varsigma, \tau \hat{\nu} v \theta \varepsilon 0 v \rho \gamma \hat{\omega} v$ : $\mathrm{K}$. se déclare dans l'incertitude quant à la provenance, mais ces doutes disparaissent chez Des Places. Enfin, K. ne se prive pas de citer in extenso des témoignages certes non 
littéraux, mais parfois d'une importance cruciale, tel celui de l'Anonyme de Turin sur le Parménide (p. 16), rejeté sans commentaire par Des Places dans les apparats des OC 3 et 26. C'est bien à la manière de K., en citant les contextes et en prenant en compte aussi les simples témoignages, qu'il faudra un jour rééditer les $O C$. On pourrait ainsi mieux prendre en compte les fragments des écrits attribuables aux théurges (voir plus bas), ainsi que les témoignages latins, dont Des Places donne un compte rendu dans son Introduction pour mieux les évincer du reste de l'édition : il ne dit pas un mot, par exemple, du fragment oraculaire que K. (p. 94) croit déceler dans le De providentia de Proclus ( $\$ 35$, p. 144 Boese).

La sûreté du jugement de K. a en outre été confirmée à maintes reprises. K. (p. 6-7) avait raison de refuser toute influence des $O C$ sur le livre de Porphyre La philosophie tirée des oracles, quoi qu'en ait dit plus tard H. Lewy. Surtout, il a identifié nettement le fait capital de la transmission des $O C$, à savoir leur nature étrangère à la philosophie des néoplatoniciens, qui se sont pourtant échinés à les interpréter (p. 11; p. 13 ; p. 45 ; p. 61), de façon parfois pratiquement indiscernable (p. 24) : ceci vaut pour

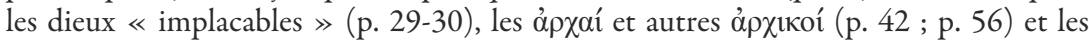
« mainteneurs » (p. 60). Un tel acharnement en dit long sur le caractère sacré revêtu par les $O C$, mais aussi sur la pauvreté des sources révélées des néoplatoniciens, contraints d'élever au pinacle un écrit sans passé et relevant d'une vision du monde différant de la leur. Il signifie également que les néoplatoniciens - qui ne se disaient jamais que «platoniciens »- n'opéraient pas de distinction fondamentale entre leurs propres vues et celles des siècles précédents, et en particulier de l'époque médio-platonicienne dont les $O C$ sont un bon témoin. On ne sait si on doit se réjouir du recul critique acquis aujourd'hui par les historiens de la philosophie, ou bien voir là un avertissement contre notre tendance à cloisonner des processus historiques par définition continus.

Le livre de K. mérite enfin d'être repris pour ses hypothèses d'un grand intérêt. $\mathrm{K}$. était sensible à la distinction entre $O C$ et écrits propres des « théurges » (p. 103) dont il suppose à plusieurs reprises une influence directe sur les néoplatoniciens (p. 23 ; p. $31 ;$ p. $46 ;$ p. $56 ;$ p. 67), notamment Jamblique (p. 8), comme le confirme entre autres la Lettre 12 de l'empereur Julien. Je crois toutefois qu'il a tort de nier la provenance jamblichéenne de Proclus, In Tim. I, 308, 17 sqq. (p. 22 ; 31). Un rapprochement intéressant est fait p. 76 n. 2 entre le De anima de Jamblique et les $O C$ à propos de la purification de l'âme par le Soleil : il faudrait mesurer s'il contredit ou non le témoignage d'Augustin, Cité de Dieu X, 23, 1 sur les $\tau \varepsilon \lambda \varepsilon \tau \alpha i ́$ du Soleil et de la Lune. On doit également à K. d'utiles conjectures philologiques (p. 68 n. 3 ; 84).

La connaissance des textes manifestée par K. stupéfie : ainsi, p. 10 (passage opportunément réintégré dans le texte par $\mathrm{S}$. depuis les Addenda), du dialogue intitulé Hermippe ou De l'astrologie, qu'il édita, et retombé depuis dans l'anonymat scientifique ; ou, p. 108, des Geoponica syriaques. De nombreuses notes évoquent le contexte des $O C$ : gnose, hermétisme, papyrus magiques, et jusqu'au judaïsme contemporain, dans la version platonisante de Philon ou dans d'autres (p. 90 ; 95). Sur ce dernier point, les intuitions de K. ont récemment été justifiées par M. Tardieu ( « Le paradis chaldaïque (fr. 107 et 165) », dans A. Lecerf, L. Saudelli, H. Seng (éd.), Oracles chaldaïques, fragments et philosophie, Heidelberg 2014, p. 15-29).

Le lecteur trouvera quelques incongruités, dont on relèvera les plus sérieuses. Relativement aux traductions : p. 11, pour « seraient au-dessus de tout soupçon », lire «me semblent au-dessus de tout soupçon ». P. 13 n. 1, pour «C'est le cas pour Proclus... », lire « Nous avons affaire à Proclus... ». Milieu de la p. 28 : il est contradictoire d'écrire que la foudre n'émousse pas le feu hypercosmique mais qu'elle s'affaiblit à 
mesure qu'elle procède : il semble qu'il faille lire dans $\mathrm{K}$. : non solum transmundani ignis

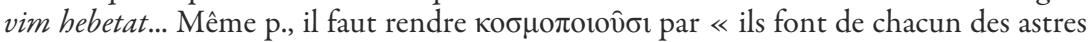
un monde... ». P. 54, dans les citations d'OC 40 et du Timée, il est dommage de traduire différemment les deux occurrences de vosivv. P. 59 n. 2 : lire « livres de Ieou ». P. 66,

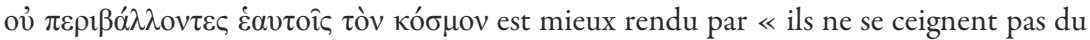
monde » (c'est-à-dire, ils ne sont pas inclus en lui). P. 73, dans la citation d'OC 109, il faut restituer la double négation (« ne reçoit pas... »); et à la p. 75, pour $O C 112$, l'impératif grec («que s'ouvre... »). P. 92, lire « lieu sublunaire » pour « dieu sublunaire ».

Voici une liste d'erreurs de référence. Bas de la p. 23, pour « p. 11-12», lire « p. 8 ». P. 29, les renvois à $O C 35$ portent sur le haut de la même page. P. 44 n. 1, lire « p. 25, fr. 27 $\mathrm{dP} \gg$. P. 62, la référence manquante est celle à Damascius qui précède immédiatement ; celle-ci à son tour devrait être substituée par «Deprinc. III, p. 117.4 ». Bas de la p. 70, la référence manquante est à la p. 68 n. 2 (l'âme comme feu).P. 93 n. 2, la dernière référence est en fait à Proclus, In Remp. II, 347.6, p. 37 de la traduction.

Quant aux remarques diverses : p. 4, il n'est pas en fait question du Baroccianus 131, manuscrit d'Oxford, mais du Vat. gr. 1026 (cité p. précédente). P. 90, dans la citation

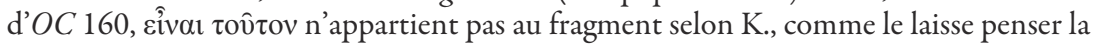
mise en page.

Ces remarques ne remettent pas en cause la grande qualité et l'utilité d'un travail pour lequel il faut adresser notre reconnaissance à $S$. ainsi qu'à ses relecteurs, C. Luna et R. Goulet. Au-delà des $O C$ et de la tradition platonicienne, on en recommande la lecture aux chercheurs se consacrant à la Gnose (K. lance l'appellation de « gnose païenne » à propos des $O C$, p. 101 ; p. 108) et à l'histoire des religions.

Adrien Lecerf

CNRS - UMR 8061, Centre Léon-Robin 